\title{
A THEORETICAL ANALYSIS OF THE ELECTRET AIR-GAP FIELD- EFFECT STRUCTURE FOR SENSOR APPLICATIONS
}

\author{
A J SPRENKELS, J A VOORTHUYZEN and P BERGVELD
}

Twente Unwersity of Technology, $P O B O x 217,7500$ AE Enschede (The Netherlands)

(Received July 30, 1985, in revised form December 6, 1985, accepted January 3, 1986)

\section{Abstract}

In order to develop a capacitive solid state sensor that makes use of an electret, a theoretical analysis is given of an electret air-gap field-effect structure

This structure is basically an MOS transistor with a movable gate and can thus be considered as a pressure-sensitive field effect transistor

It is shown that the addition of a metal layer on top of the semiconductor-oxide increases the sensitivity due to charge density multiplication

All calculations are based upon the displacement sensitivity $S$, which is independent of the mechanical properties of the diaphragm and thus independent of a specific application

Based upon the calculated sensitivities of the several configurations, a well-considered decision can be made as to which configuration is best suited for a specific application In this paper this has been done for a solid state microphone and a pressure sensor as examples

\section{Introduction}

Pressure sensors based on the capacitive principle recelve considerable attention nowadays [ $1-5]$ The reasons for this interest are the advantages of this type of sensor compared to, for instance, sensors based on the piezoresistive principle [5 - 8]

A classic example of a capacitive sensor is the well-known condenser microphone, which combines a relatively high sensitivity with an extremely flat frequency response over a range of several decades [9] A more recent example is the capacitive pressure sensor, which exhibits a good linearity by using a special electronic crrcuit [4] and a sensitivity about ten times higher than the corresponding piezoresistive devices [5]

A drawback of the capacitive sensor is, however, the fact that rather small capacitance variations must be detected This requires a relatively complex detection circuitry $[1,5]$ Since the MOS transistor is an inherently capacitive device, the combination of a capacitive sensor and an MOS transistor seems quite obvious Integration of the capacitive sensor and an 
$n$-channel MOS transistor results in a field-effect device having a movable gate electrode, separated from the oxide layer by a small aur gap as shown in Fig 1 Basically this configuration has been described by several authors $[10,11]$

By fabricating the capacitive mechanical part in the same process as the MOS transistor, advantage can be taken of all the possibilities of IC technology, such as micro-machining and photolithographic processes This can lead to batch fabrication with low production costs per sensor, a better reproducibility and the possibility of on-chip electronic circuitry

Using IC technology, practical values of the air-gap space are of the order of several microns As a consequence the thickness of the layered dielectme of the modified MOS transistor in Fig 1 is much larger than the oxide thickness of a conventional MOS transistor Since the sensitivity is proportional to the field strength in the air gap, a rather high gate voltage will be necessary in order to obtain a competitive sensitivity with respect to other pressure sensors This can be accomplished either by an external voltage source (cf, condenser microphone), or by the addition of an internal permanently charged polymer (electret) to this system (cf, electret microphone) This results in the basic configuration as presented in Fig 2 and basically described in ref. 12

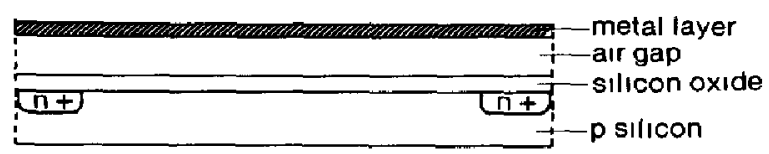

Fig 1 MOS transistor with a movable gate

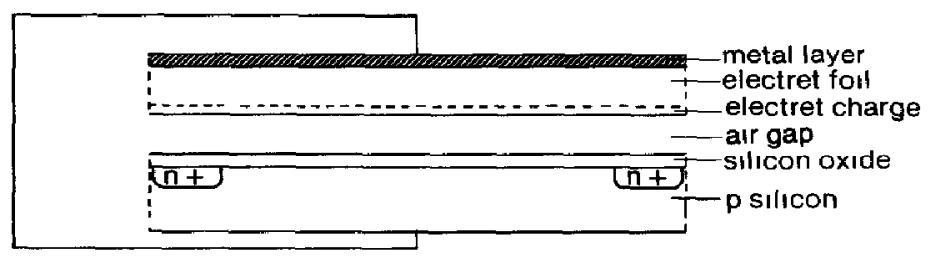

Fig 2 Basic configuration of an electret air-gap field-effect structure

In this structure the dielectric of the gate capacitance consists of an oxide layer, an air gap and an electret foil In principle it is an MOS transistor with a movable gate or diaphragm, consisting of a metallized electret The fixed charge of the electret induces an equal amount of charge of opposite sign in the upper metal layer and in the channel of the MOS transistor The distribution of this charge between the upper metal and the channel depends on the thickness of the electret foll and the air gap Pressure variations, acting on the diaphragm, change the alr-gap distance and thus the air-gap capacitance, which is accompanied by a redistribution of charge between the upper metal layer and the channel This charge transfer results in a change of the channel conductance, which can be measured In this way 
the MOS transistor, having a compound dielectric, has become pressure sensitive

This principle of operation is applicable to different types of pressuresensing devices such as a microphone and a blood pressure sensor This implies that mechanical design considerations, concerning for instance diaphragm properties and air damping, depend to a great extent on the specific application and will therefore not be considered In this paper we will only consider the common aspects of possible sensor applications

Therefore, it is adequate to introduce a parameter $S$, which is defined as the change of the drain current $I_{\mathrm{d}}$ due to a change in the arr-gap distance $s_{\mathbf{a}}$

$S=\frac{\mathrm{d} I_{\mathrm{d}}}{\mathrm{d} s_{\mathrm{a}}}$

In accordance with Yoshikawa et al [11] we will refer to this parameter $S$ as the displacement sensitivity or in short 'sensitivity' Since this parameter is independent of the way in which $\mathrm{d} s_{\mathrm{a}}$ is realized and thus independent of a specific application, it provides an opportunity to optimize the electrical part of the sensor

Considering Fig 2, it will be clear that the electret foll and the air gap can be interchanged In that case we obtain the structure shown in Fig 3 This metal arr electret oxide silicon (MAEOS) structure provides several advantages as compared to the metal electret air oxide silicon (MEAOS) structure

First of all it should be noted that those materials best suited for electret applications, such as Teflon, have rather poor mechanical properties Therefore, applying the MAEOS structure the diaphragm material as well as the electret material can be chosen freely and optimized separately

A second advantage of the MAEOS structure as compared to the MEAOS structure is the passivation of the silicon oxide by a Teflon layer, which appears to be very inert In this way the instabilities, as reported by Yoshikawa et al [11], caused by changes at the oxide-air interface, can be reduced

In this paper a theoretical analysis will be given of the basic configuration as shown in Fig 3 and two modifications thereof Contrary to Yoshikawa et $a l$, we will assume that the field-effect structure operates in the non-saturated mode, which results in a less complicated and therefore more useful theoretical description

It will appear that the sensitivities $S$ of these three configurations differ Based upon the results, a well-considered decision can be made as to which configuration is best suited for several realizations

\section{The MAEOS configuration}

The MAEOS configuration ( $\mathrm{F} 1 \mathrm{~g} 3$ ) consists of an electret foil, attached drrectly to the oxıde layer, an air gap and a separate conductıng diaphragm 


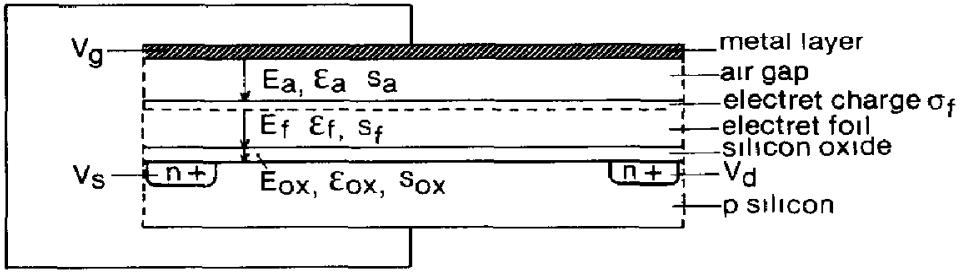

Fig 3 The MAEOS configuration

The electrical field strength in the three insulators is denoted as $E_{\jmath}$, the relative dielectric constant as $\epsilon_{j}$ and the thickness as $s_{j}$ A constant surface charge per unit area $Q_{s}$, accounting for the fixed oxide charge as well as fixed interface states, is assumed to be present at the $\mathrm{Si}_{1}-\mathrm{SiO}_{2}$ interface, while the channel charge per unit area and the depletion charge per unit area are denoted as $Q_{c}$ and $Q_{d}$ respectively Using Gauss's theorem, three expressions for the voltage across the insulators can be derived

$$
\begin{aligned}
& E_{\mathrm{a}} s_{\mathrm{a}}=\frac{-1}{C_{\mathrm{a}}^{\mathrm{D}}}\left(Q_{\mathrm{d}}+Q_{\mathrm{c}}+Q_{\mathrm{s}}\right)-V_{\mathrm{a}} \\
& E_{\mathrm{f}} s_{\mathrm{f}}=\frac{-1}{C_{\mathrm{f}}{ }^{\mathrm{D}}}\left(Q_{\mathrm{d}}+Q_{\mathrm{c}}+Q_{\mathrm{s}}\right) \\
& E_{\mathrm{ox}} s_{\mathrm{ox}}=\frac{-1}{C_{\mathrm{ox}}}\left(Q_{\mathrm{d}}+Q_{\mathrm{c}}+Q_{\mathrm{s}}\right)
\end{aligned}
$$

where $V_{\mathrm{a}}=\sigma_{\mathrm{f}} / C_{\mathrm{a}}$, with $\sigma_{\mathrm{f}}$ the electret charge per unit area assumed to be present at the electret-air interface and $C$, the capacitance per unit area for the three insulators respectively

Defining the potential of the channel with respect to the substrate as $V(x)$ and assuming that a channel exists, the gate voltage with respect to the substrate can be written as

$$
V_{\mathrm{g}}=V(x)+2 \phi_{\mathrm{f}}+\phi_{\mathrm{ms}}+E_{\mathrm{ox}} s_{\mathrm{ox}}+E_{\mathrm{f}} s_{\mathrm{f}}+E_{\mathrm{a}} s_{\mathrm{a}}
$$

where $\phi_{\mathrm{f}}$ is the Fermi potential difference between doped bulk silicon and intrinsic silicon and $\phi_{\mathrm{ms}}$ the metal-silicon Fermi potential difference The following expression for the charge in the channel is obtained from eqns (1) and (2)

$Q_{\mathrm{c}}=-C_{\mathrm{t}}\left[V_{\mathrm{g}}+V_{\mathrm{a}}-2 \phi_{\mathrm{f}}-\phi_{\mathrm{ms}}-V(x)\right]-Q_{\mathrm{d}}-Q_{\mathrm{s}}$

where

$$
C_{\mathrm{t}}{ }^{\mathrm{D}}=\frac{C_{\mathrm{a}}{ }^{\square} C_{\mathrm{f}}{ }^{\mathrm{D}} C_{\mathrm{ox}}}{C_{\mathrm{a}}{ }^{\square} C_{\mathrm{f}}{ }^{\square}+C_{\mathrm{a}}{ }^{\square} C_{\mathrm{ox}}{ }^{\square}+C_{\mathrm{f}}{ }^{\square} C_{\mathrm{ox}}{ }^{\square}}
$$


The non-saturated drain current can be written as described in ref 13

$I_{\mathrm{d}}=-\mu \frac{W}{L} \int_{V_{\mathrm{s}}}^{V_{\mathrm{d}}} Q_{\mathrm{c}}(V) \mathrm{d} V$

where $W$ and $L$ are the channel width and length respectively and $\mu$ is the mobility of the charge carriers in the channel

Substituting eqn (3) into eqn (5), and assuming $Q_{d}$ is constant, the following drain-current expression results.

$I_{\mathrm{d}}=\mu \frac{W}{L}\left[C_{\mathrm{t}}^{\square} V_{\mathrm{a}}^{\prime} V_{\mathrm{ds}}+Q_{\mathrm{s}} V_{\mathrm{ds}}+Q_{\mathrm{d}} V_{\mathrm{ds}}\right]$

where

$V_{\mathrm{a}}^{\prime}=V_{\mathrm{a}}+V_{\mathrm{gs}}-\frac{V_{\mathrm{ds}}}{2}-2 \phi_{\mathrm{f}}-\phi_{\mathrm{ms}}$

$\Lambda$ ssuming that the drain-source voltage $V_{\mathrm{ds}}$ is kept constant, the sensitivity $S$, which has been defined as $S=\mathrm{d} I_{\mathrm{d}} / \mathrm{d} s_{\mathrm{a}}$, can be found by differentiating eqn (6) with respect to $s_{\mathrm{a}}$ and using the relations $C_{\mathrm{a}}{ }^{\square}=\epsilon_{0} / s_{\mathrm{a}}$ and $V_{\mathrm{a}}=\sigma_{\mathrm{f}} / C_{\mathrm{a}}{ }^{\square}$ We thus obtain

$S=\mu \frac{W}{L} \frac{V_{\mathrm{ds}}}{s_{\mathrm{a}}} C_{\mathrm{a}}{ }^{\square} C_{\mathrm{f}}{ }^{\square} C_{\mathrm{ox}}\left[\frac{V_{\mathrm{a}}\left(C_{\mathrm{a}}{ }^{\square} C_{\mathrm{ox}}{ }^{\square}+C_{\mathrm{a}}{ }^{\square} C_{\mathrm{f}}{ }^{\square}\right)+\left(V_{\mathrm{a}}-V_{\mathrm{a}}{ }^{\prime}\right) C_{\mathrm{f}}{ }^{\square} C_{\mathrm{ox}}{ }^{\square}}{\left(C_{\mathrm{a}}{ }^{\square} C_{\mathrm{f}}{ }^{\square}+C_{\mathrm{a}}{ }^{\square} C_{\mathrm{ox}}{ }^{\square}+C_{\mathrm{f}}{ }^{\square} C_{\mathrm{ox}}{ }^{\square}\right)^{2}}\right]$

However, in practical situations the thicknesses of the electret and the air gap are several microns, while the oxide thickness is about one tenth of a micron, ie $C_{\mathrm{ox}}{ }^{\square} \gg C_{\mathrm{a}}{ }^{\square}$ and $C_{\mathrm{f}}{ }^{\mathrm{D}}$ In addition, the voltage $V_{\mathrm{a}}=\sigma_{\mathrm{f}} / C_{\mathrm{a}}{ }^{\mathrm{D}}$ is much larger than $2 \phi_{\mathrm{f}}, \phi_{\mathrm{ms}}, V_{\mathrm{gs}}$ and $V_{\mathrm{ds}}$, since the transistor is assumed to be nonsaturated

Thus expression (8) can be approxımated by

$S=\mu \frac{W}{L} \frac{V_{\mathrm{ds}}}{s_{\mathrm{a}}} \sigma_{\mathrm{f}} \frac{C_{\mathrm{a}}{ }^{\mathrm{D}} C_{\mathrm{f}}{ }^{\square}}{\left(C_{\mathrm{a}}{ }^{\mathrm{D}}+C_{\mathrm{f}}{ }^{\mathrm{D}}\right)^{2}}$

For a given value of $s_{\mathrm{a}}$, and thus $C_{\mathrm{a}}$, optimum sensitivity is obtained if $C_{\mathrm{f}}{ }^{\mathrm{D}}=C_{\mathrm{a}}{ }^{\mathrm{D}}$, which yields

$S_{\text {opt }}=\mu \frac{W}{L} \frac{V_{\mathrm{ds}} \sigma_{\mathrm{f}}}{s_{\mathrm{a}} 4}$

The condition $C_{\mathrm{f}}{ }^{\square}=C_{\mathrm{a}}{ }^{\square}$ can easily be satisfied by choosing an appropriate value for the thickness $s_{\mathrm{f}}$ of the electret foll $s_{\mathrm{f}}=\epsilon_{\mathrm{f}} s_{\mathrm{a}}$

\section{Increased sensitivity}

In the previous section the areas of the capacitances $C_{\mathrm{a}}, C_{\mathrm{f}}$ and $C_{\mathrm{ox}}$ were assumed to be equal or, in other words, the channel area determined the 
dimensions of the oxide, the aur-gap and electret capacitances As previously mentioned, a reduction of the total charge on the upper metal layer is accompanied by an equal increase of the total charge in the channel of the MOS transistor If the areas of all capacitances are equal, the changes of charge per unit area on the upper metal layer and the channel are also equal to each other By depositing a metal layer on top of the oxide surface, which is larger than the gate area $W L$, the area of the diaphragm, and consequently $C_{\mathrm{a}}$ and $C_{\mathrm{f}}$, is also increased This implies an increased change of the total charge on the upper metal layer and thus an increased change of the channel charge $Q_{\mathrm{c}}$ per unit area. The drain current $I_{\mathrm{d}}$ of the MOS transistor depends linearly on the channel charge $Q_{c}$ per unit area, so the introduction of a metal layer, which is larger than the gate area $W L$, on top of the oxide surface results in a higher sensitivity due to charge-density multiplication

On the other hand, it should be noted that by the addition of a metal layer on top of the oxide layer, parasitic capacitances in parallel with the oxide capacitance are introduced, which in turn reduce the sensitivity Nevertheless, it can be shown that the addition of a metal layer always increases the sensitivity as long as the thickness of the gate oxide is considerably less than that of the so-called field oxide above the surrounding substrate In practical situations this is always the case With this additional floating metal layer, the MAEOS structure is modified to the metal air electret metal oxide silicon (or MAEMOS) configuration, which is shown in Fig 4 In order to obtain an expression for the sensitivity $S$, a more straightforward procedure will be followed as basically outlined in ref 12 This configuration will be considered as a combination of a conventional MOS transistor and an electret air-gap structure, which is schematically shown in Fig 5

It consists of four capacitances with unequal areas The capacitance $C_{p}$ represents the total parasitic capacitance connected with the gate of the

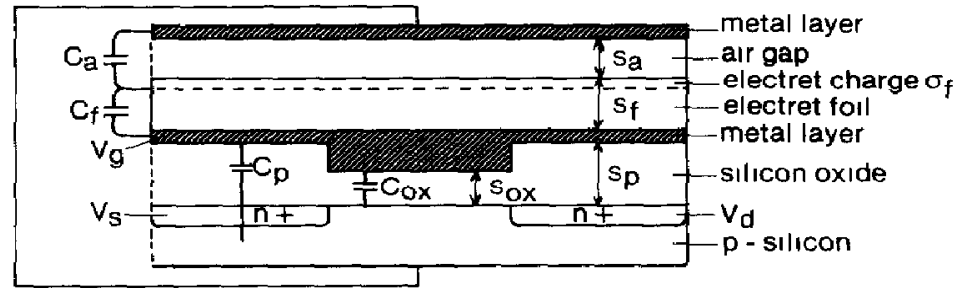

Fig 4 The MAEMOS configuration

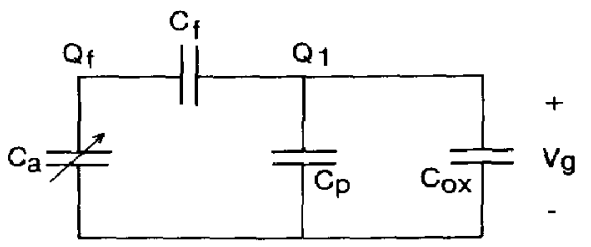

Fig 5 Schematic representation of the MAEMOS configuration 
MOS transistor If the area of the metal layer on top of the oxide surface, and thus of $C_{\mathrm{a}}$ and $C_{\mathrm{f}}$, is equal to $A$, and $s_{\mathrm{p}}$ is the thickness of the field oxide, the following equations for the capacitances are valid

$$
\begin{array}{ll}
C_{\mathrm{a}}=\frac{\epsilon_{0} A}{s_{\mathrm{a}}} & C_{\mathrm{f}}=\frac{\epsilon_{0} \epsilon_{\mathrm{f}} A}{s_{\mathrm{f}}} \\
C_{\mathrm{ox}}=\frac{\epsilon_{0} \epsilon_{\mathrm{ox}} W L}{s_{\mathrm{ox}}} & C_{\mathrm{p}}=\frac{\epsilon_{\mathrm{o}} \epsilon_{\mathrm{ox}}}{s_{\mathrm{p}}}(A-W L)
\end{array}
$$

In order to be able to compare this configuration with the MAEOS configuration, we assume that no net charge $Q_{1}$ is present at the oxide-arr interface In this case the gate voltage $V_{\mathrm{g}}$ can be expressed as

$$
V_{\mathrm{g}}=\frac{\sigma_{\mathrm{f}} A C_{\mathrm{f}}}{C_{\mathrm{a}} C_{\mathrm{f}}+C_{\mathrm{a}} C_{1}+C_{\mathrm{f}} C_{1}}
$$

where

$C_{1}=C_{\mathrm{p}}+C_{\mathrm{ox}}$

This results in the following expression for the gate-voltage variations, due to variations in the arr-gap distance $s_{\mathrm{a}}$

$\frac{\mathrm{d} V_{\mathrm{g}}}{\mathrm{d} s_{\mathrm{a}}}=\frac{\sigma_{\mathrm{f}} A C_{\mathrm{a}} C_{\mathrm{f}}\left(C_{1}+C_{\mathrm{f}}\right)}{\left(C_{\mathrm{a}} C_{\mathrm{f}}+C_{\mathrm{a}} C_{1}+C_{\mathrm{f}} C_{1}\right)^{2}} \frac{1}{s_{\mathrm{a}}}$

In first-order MOS theory, the expression for the drain current in the nonsaturated region is

$I_{\mathrm{d}}=\mu \frac{W}{L} C_{\mathrm{ox}}\left[\left(V_{\mathrm{g}}-V_{\mathrm{T}}\right) V_{\mathrm{ds}}-\frac{1}{2} V_{\mathrm{ds}}{ }^{2}\right]$

where $V_{\mathrm{T}}$ is the threshold voltage [13]

Assuming that $V_{\mathrm{ds}}$ is kept constant, the variation of the dram current due to variations in the aur-gap distance can be written as

$\frac{\mathrm{d} I_{\mathrm{d}}}{\mathrm{d} s_{\mathrm{a}}}=\mu \frac{W}{L} C_{\mathrm{ox}}{ }^{\square} V_{\mathrm{ds}} \frac{\mathrm{d} V_{\mathrm{g}}}{\mathrm{d} s_{\mathrm{a}}}$

Substituting eqn (14) into eqn (16) and applying eqns (11), the sensitivity $S$ can be expressed as

$S=\mu \frac{W}{L} \frac{V_{\mathrm{ds}}}{s_{\mathrm{a}}} \frac{\sigma_{\mathrm{f}}}{4} \frac{4 A C_{\mathrm{a}}{ }^{\square} C_{\mathrm{f}}{ }^{\square} C_{\mathrm{ox}}{ }^{\square}\left[C_{\mathrm{p}}{ }^{\square}(A-W L)+W L C_{\mathrm{ox}}{ }^{\square}+A C_{\mathrm{f}}{ }^{\square}\right]}{\left\{A C_{\mathrm{a}}{ }^{\square} C_{\mathrm{f}}{ }^{\square}+\left[C_{\mathrm{a}}{ }^{\square}+C_{\mathrm{f}}{ }^{\square}\right]\left[C_{\mathrm{p}}{ }^{\square}(A-W L)+W L C_{\mathrm{ox}}{ }^{\square}\right]\right\}^{2}}$

If $s_{\mathrm{p}} \gg s_{\mathrm{ox}}$ the influence of the parasitic capacitance cancels and optimum sensitivity is achieved In this case eqn (17) reduces to 
$S=\mu \frac{W}{L} \frac{V_{\mathrm{ds}}}{s_{\mathrm{a}}} \frac{\sigma_{\mathrm{f}}}{4} \frac{4 A C_{\mathrm{a}}{ }^{\square} C_{\mathrm{f}}^{\square} C_{\mathrm{ox}}\left(A C_{\mathrm{f}}^{\square}+W L C_{\mathrm{ox}}^{\square}\right)}{\left[A C_{\mathrm{a}}^{\square} C_{\mathrm{f}}^{\square}+\left(C_{\mathrm{a}}^{\square}+C_{\mathrm{f}}^{\square}\right) W L C_{\mathrm{ox}}\right]^{2}}$

which has an optımum value if

$A \gg C_{\mathrm{ox}}{ }^{\triangleright} W L\left(\frac{C_{\mathrm{a}}{ }^{\square}+C_{\mathrm{f}}{ }^{\square}}{C_{\mathrm{a}}{ }^{\square} C_{\mathrm{f}}{ }^{\square}}\right)$

Applying this condition, eqn (18) reduces to

$S_{\mathrm{opt}}=\mu \frac{W}{L} \frac{V_{\mathrm{ds}}}{s_{\mathrm{a}}} \frac{\sigma_{\mathrm{f}}}{4} \frac{4 C_{\mathrm{ox}}{ }^{\mathrm{D}}}{C_{\mathrm{a}}{ }^{\mathrm{a}}}$

Comparing eqns (10) and (19), we note that the sensitivity of the MAEMOS structure is considerably larger than the sensitivity of the MAEOS structure, because the oxide capacitance $C_{\text {ox }}$ per unit area is much larger than the aurgap capacitance $C_{\mathrm{a}}$ per unit area

\section{A c operation}

In the previous Section it has been shown that the addition of a metal layer on top of the oxide layer in order to extend the effectrve gate area improves the sensitivity considerably However, a metal layer on top of the oxide layer has another advantage as well, as will now be outlined

In order to be able to compare the MAEMOS and the MAEOS structures, we assumed in Section 3 that the charge $Q_{1}$ was zero However, in a practical realization this condition cannot be guaranteed easily, since all kinds of charge transport over the oxide surface may occur This problem is solved if the oxide surface has a well-defined potential with respect to the bulk This can be accomplished by connecting a resistance between the metal layer on top of the oxide, and the bulk of the device, which forces the voltage across the oxide layer to be zero However, this resistance determines, in conjunction with the capacitances $C_{\mathrm{a}}, C_{\mathrm{f}}$ and $C_{1}$, the low-frequency cut-off of the sensor As a consequence only a $c$ pressure variations can be measured This low-frequency cut-off can be as low as $10^{-3} \mathrm{~Hz}$ by an appropriate choice of the value of the resistance [14]

Th1s modified MAEMOS structure will be called the MAEMOS-R configuration and is schematically represented in $F_{1 g} 6$ The gate voltage $V_{\mathrm{g}}$ can be written as

$V_{\mathrm{g}}=\frac{Q_{\mathrm{f}} C_{\mathrm{f}}+\left(C_{\mathrm{a}}+C_{\mathrm{f}}\right) Q_{\mathrm{t}}}{C_{\mathrm{a}} C_{\mathrm{f}}+C_{\mathrm{a}} C_{\mathrm{i}}+C_{\mathrm{f}} C_{\mathrm{l}}}$

The time constant $\tau$ of the system, which determines the low-frequency cutoff, can be written as 


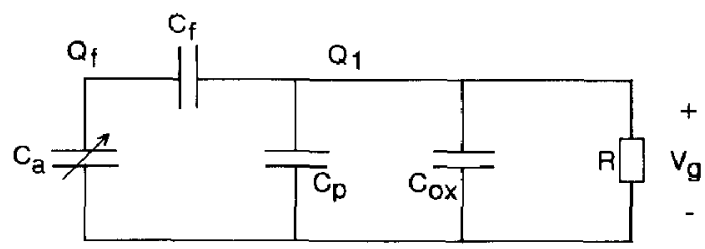

Fig 6 Schematic representation of the MAEMOS-R configuration

$\tau=R\left(C_{1}+\frac{C_{\mathrm{a} 0} C_{\mathrm{f}}}{C_{\mathrm{a} 0}+C_{\mathrm{f}}}\right)$

with $C_{\mathrm{a} 0}$ the value of $C_{\mathrm{a}}$ for zero pressure

The expression for the gate voltage variation due to air-gap variations, which are fast compared to the time constant $\tau$, has the following form

$\frac{\mathrm{d} V_{\mathrm{g}}}{\mathrm{d} s_{\mathrm{a}}}=\frac{\left(C_{\mathrm{a}} C_{\mathrm{f}}^{2}+C_{\mathrm{a}} C_{\mathrm{f}} C_{\mathrm{1}}\right) Q_{\mathrm{f}}+C_{\mathrm{a}} C_{\mathrm{f}}^{2} Q_{1}}{\left(C_{\mathrm{a}} C_{\mathrm{f}}+C_{\mathrm{a}} C_{1}+C_{\mathrm{f}} C_{1}\right)^{2}} \frac{1}{s_{\mathrm{a}}}$

Assuming that the ' $\mathrm{d} \mathrm{c}$ gate voltage' $V_{\mathrm{g}}$ is zero if no pressure is applied to the diaphragm, the charge $Q_{1}$ can be written as

$Q_{1}=\frac{-Q_{\mathrm{f}} C_{\mathrm{f}}}{C_{\mathrm{a} 0}+C_{\mathrm{f}}}$

Substituting eqn (23) in eqn (22) and assuming that variations of the airgap capacitance around the zero pressure value are small, $l e, C_{\mathrm{a} 0}=C_{\mathrm{a}}$, expression (22) reduces to

$\frac{\mathrm{d} V_{\mathrm{a}}}{\mathrm{d} s_{\mathrm{a}}}=\frac{\sigma_{\mathrm{f}} A C_{\mathrm{a}} C_{\mathrm{f}}}{\left(C_{\mathrm{a}}+C_{\mathrm{f}}\right)\left(C_{\mathrm{a}} C_{\mathrm{f}}+C_{\mathrm{a}} C_{1}+C_{\mathrm{f}} C_{1}\right)} \frac{1}{s_{\mathrm{a}}}$

Using eqns (11), (16) and (24), this results in the following expression for the sensitivity

$S=\mu \frac{W}{L} \frac{V_{\mathrm{ds}}}{s_{\mathrm{a}}} \frac{\sigma_{\mathrm{f}}}{4} \frac{4 A C_{\mathrm{a}}{ }^{\square} C_{\mathrm{f}}{ }^{\square} C_{\mathrm{ox}}{ }^{\square}}{\left(C_{\mathrm{a}}{ }^{\square}+C_{\mathrm{f}}{ }^{\square}\right)\left\{A C_{\mathrm{a}}{ }^{\square} C_{\mathrm{f}}^{\square}+\left[C_{\mathrm{a}}^{\square}+C_{\mathrm{f}}{ }^{\square}\right]\left[C_{\mathrm{p}}{ }^{\square}(A-W L)+W L C_{\mathrm{ox}}{ }^{\square}\right]\right\}}$

This expression has an optimum value if $s_{\mathrm{p}} \gg s_{\mathrm{ox}}$ and $A \gg C_{\mathrm{ox}}{ }^{\square} W L\left(C_{\mathrm{a}}{ }^{\square}+C_{\mathrm{f}}{ }^{\mathrm{D}}\right) /$ $C_{\mathrm{a}}{ }^{\square} C_{\mathrm{f}}{ }^{\mathrm{D}}$

Under these assumptions eqn (25) reduces to

$S_{\mathrm{opt}}=\mu \frac{W}{L} \frac{V_{\mathrm{ds}}}{s_{\mathrm{a}}} \frac{\sigma_{\mathrm{f}}}{4} \frac{4 C_{\mathrm{ox}}{ }^{\square}}{\left(C_{\mathrm{a}}^{\square}+C_{\mathrm{f}}{ }^{\mathrm{D}}\right)}$

Considering eqns (26) and (19), we note that the sensitivity of the MAEMOS-R structure is about a factor two smaller than the sensitivity of 
the MAEMOS structure, assuming that the air-gap capacitance $C_{a}$ per unit area and the electret capacitance $C_{\mathrm{f}}$ per unit area are equal to each other

\section{Theoretical results}

We have derived the expressions for the sensitivity, as defined in Section 1, for three different electret arr-gap sihcon structures These three expressions are summarized below for comparison All sensitivities are expressed in Lerms of $K$, which is the sensitivity of the MAEOS configuration as described in Section 2

MAEOS

$$
S=\mu \frac{W}{L} \frac{V_{\mathrm{ds}}}{s_{\mathrm{a}}} \frac{\sigma_{\mathrm{f}}}{4}=K
$$

MAEMOS $S=K \frac{4 A C_{\mathrm{a}}{ }^{\circ} C_{\mathrm{f}}{ }^{\square} C_{\mathrm{ox}}{ }^{\square}\left[C_{\mathrm{p}}(A-W L)+W L C_{\mathrm{ox}}{ }^{\square}+A C_{\mathrm{f}}{ }^{\square}\right]}{\left\{A C_{\mathrm{a}}{ }^{\square} C_{\mathrm{f}}{ }^{\square}+\left[C_{\mathrm{a}}{ }^{\square}+C_{\mathrm{f}}{ }^{\square}\right]\left[C_{\mathrm{p}}{ }^{\square}(A-W L)+W L C_{\mathrm{ox}}{ }^{\square}\right]\right\}^{2}}$

MAEMOS-R

$$
-K=\frac{4 A C_{\mathrm{a}}{ }^{\square} C_{\mathrm{f}}{ }^{\square} C_{\mathrm{ox}}}{\left(C_{\mathrm{a}}{ }^{\square}+C_{\mathrm{f}}{ }^{\square}\right)\left\{A C_{\mathrm{a}}{ }^{\square} C_{\mathrm{f}}{ }^{\square}+\left[C_{\mathrm{a}}{ }^{\square}+C_{\mathrm{f}}{ }^{\square}\right]\left[C_{\mathrm{p}}{ }^{\square}(A-W L)+W L C_{\mathrm{ox}}{ }^{\square}\right]\right\}}
$$

The calculated results of the sensitivities of the MAEMOS and the MAEMOS$R$ configurations are presented in Figs 7 and 8 respectively as a function of the area $A$ of the metal layer on top of the oxide layer and with the thickness $s_{\mathrm{p}}$ of the field oxide as a parameter

The following values for the different parameters are used in all calculations

$$
\begin{array}{ll}
s_{\mathrm{a}}=10 \mu \mathrm{m} & W=500 \mu \mathrm{m} \\
s_{\mathrm{f}}=20 \mu \mathrm{m} & L=10 \mu \mathrm{m} \\
s_{\mathrm{ox}}=01 \mu \mathrm{m} &
\end{array}
$$

Considering Figs 7 and 8 , the optimum sensitivities of the MAEMOS and the MAEMOS-R configurations are reached for relatively high values of $s_{\mathrm{p}}$ (approximately $s_{\mathrm{p}}=1000 \mu \mathrm{m}$ ) and for high values of $A$ (approximately $A=05 \mathrm{~cm}^{2}$ )

Nevertheless the sensitivity of the MAEMOS configuration is always higher than that of the MAEMOS-R configuration, if all parameters are the same in both cases

Using the above-mentioned values of the parameters $s_{\mathrm{a}}, s_{\mathrm{f}}, s_{\text {ox }}, W$ and $L$, and for $s_{\mathrm{p}} \rightarrow \infty$ and $A=A_{\mathrm{opt}}$, the following optimum values for the sensitivities can be calculated 

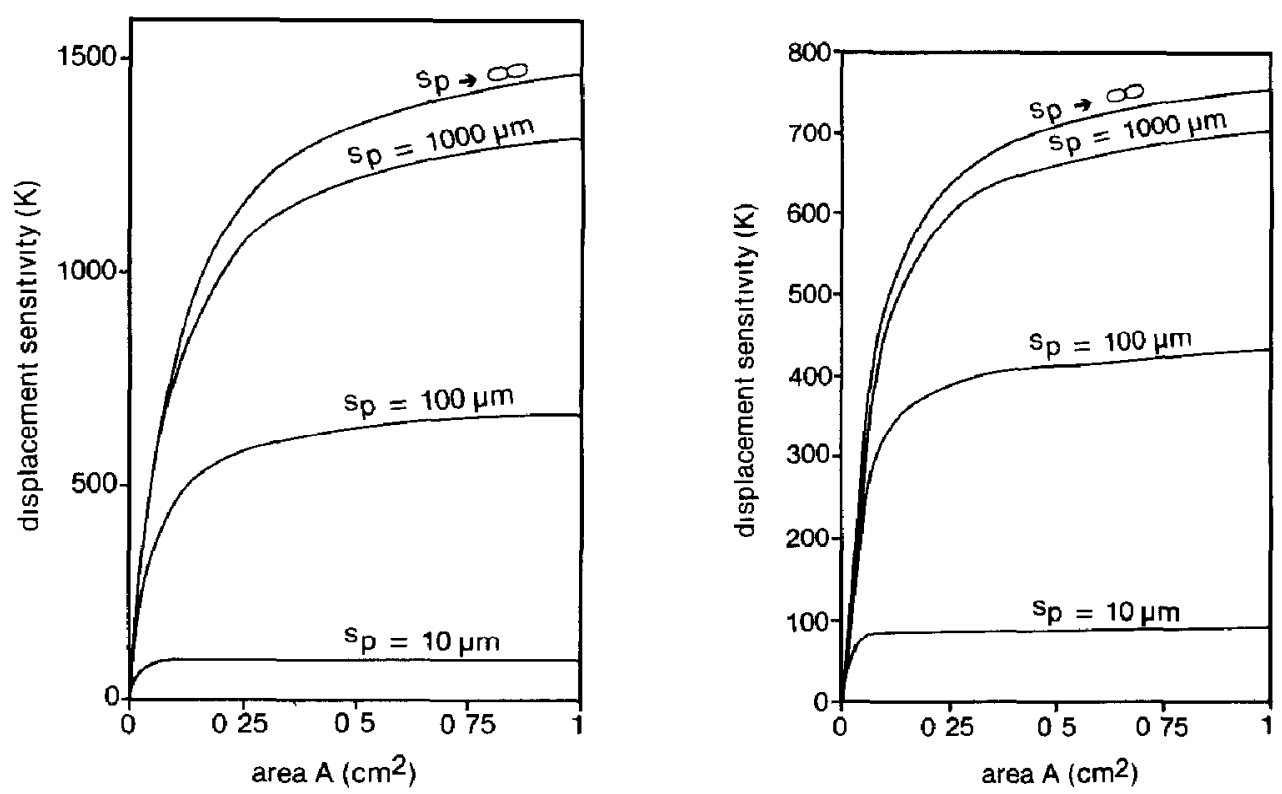

Fig 7 The calculated displacement sensitivity of the MAEMOS configuration as a function of the area $A$ with $s_{p}$ as a parameter

Fig 8 The calculated displacement sensitivity of the MAEMOS-R configuration as a function of the area $A$ with $s_{p}$ as a parameter

MAEOS $S=K$

MAEMOS $S=K \frac{4 C_{\text {ox }}{ }^{\square}}{C_{\mathrm{a}}{ }^{\square}}=1600 K$

MAEMOS-R $S=-K \frac{4 C_{\mathrm{ox}}{ }^{\square}}{C_{\mathrm{a}}{ }^{\circ}+C_{\mathrm{f}}{ }^{\mathrm{D}}}=-800 \mathrm{~K}$

Considering Figs 7 and 8 , we note that these optimum values of the sensitivity are almost reached for $s_{\mathrm{p}}=1000 \mu \mathrm{m}$ It will be clear that this high value of $s_{\mathrm{p}}$ can hardly be reached by technological means In our opinion, however, the effective value of $s_{\mathrm{p}}$ can be increased, for example to $1000 \mu \mathrm{m}$ in a final realization, by using appropriate bootstrapping techniques These optimum sensitivities are reached for relatively large values of the area $A$ Thus, if a large diaphragm area of the sensor is not a limiting factor, the best choice is the MAEMOS configuration, since this configuration exhibits the highest sensitivity

If only a $c$ pressure variations are to be measured, the MAEMOS-R configuration is preferable, since the oxide-surface potential is well defined, which results in a guaranteed stability

However, if the diaphragm area appears to be a limiting factor, let us say the maximum allowable sensor area is $2 \mathrm{~mm}^{2}$, the sensitivities of the MAEMOS and the MAEMOS-R structure appear to be almost the same, as is 


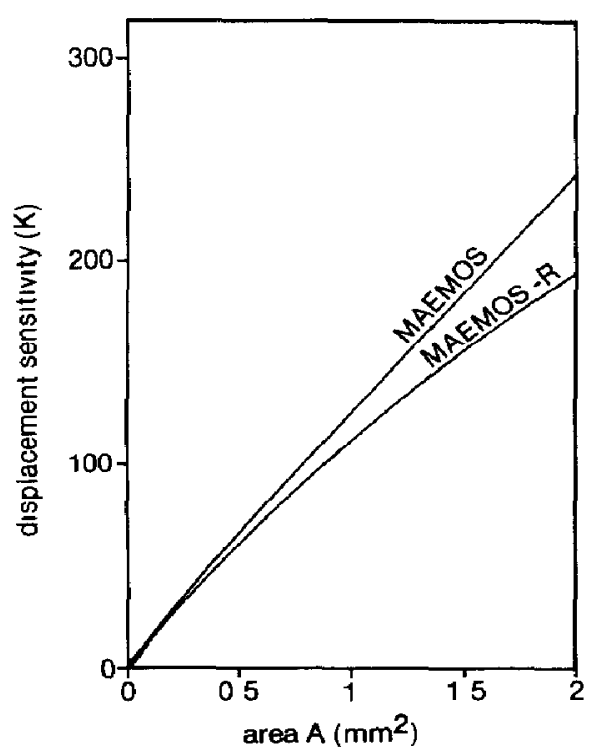

Fig 9 The calculated displacement sensitivities of two configurations as a function of the area $A$ with $s_{\mathrm{p}} \rightarrow \infty$

shown in Fig 9 In the case of a small sensor other, for instance technological, considerations will determine the choice of the configuration best suited for a specific application

\section{Conclusion and discussion}

A theoretical analysis of an electret air-gap field-effect structure has been given, which is basically an MOS transistor with a movable gate This basic structure has already been described by Yoshikawa et al [11], assuming the MOS transistor to operate in the saturated mode However, we have analysed this basic structure as well as two modifications thereof assuming that the MOS transistor operates in the non-saturated mode, which results in a less complicated and therefore more useful theoretical description

It has been shown that the addition of a metal layer, larger than the gate area, on top of the semiconductor oxide results in a charge-density multiplication and consequently in a considerable increase in the sensitivity of the sensor

All calculations are based upon the displacement sensitivity $S$, which is independent of the mechanical properties of the diaphragm and thus independent of a specific application

In particular, the definition of the displacement sensitivity $S$ has enabled us to derive three expressions upon which a well-considered decision can be made as to which configuration is best suited for several applications such as a solid state microphone and a pressure sensor, which are being investigated in our research group 
For the application as a microphone the MAEMOS- $R$ configuration is preferable The resistance across the oxide layer forces the oxide surface potential to be zero, which results in a guaranteed stability However, this resistance determines the low frequency cut-off of the sensor, which is not a limiting factor since only a c pressure variations are to be measured

The application as a pressure sensor requires that static pressures are to be measured Therefore only the MAEOS and MAEMOS configurations can be used. Which configuration is best suited for a specific pressure sensor strongly depends on the desired pressure sensitivity and pressure range

\section{Acknowledgement}

The authors wish to thank Prof O W Memelink and Dr $\mathrm{H}$ Wallinga for their stimulating discussions and helpful suggestions

These investigations in the program of the Foundation for Fundamental Research on Matter (FOM) have been supported in part by the Foundation for Technical Research (STW)

\section{References}

1 W H Ko, B -X Shao, C D Fung, W -J Shen and G -J Yeh, Sensors and Actuators, 4 (1983) $403-411$

2 Y S Lee and K D Wise, IEEE Trans Electron Devices, ED-29 (1982) 42 - 48

3 Y E Park and K D Wise, IEEE 1983 Custom Integrated Ctrcutts Conf, Rochester, $N Y, U S A$, pp $380-384$

4 T Ishida, M Kanno and O Makıno, Proc 2nd Sensor Symp, Tsukuba Sclence City, 27-28 May, 1982, pp $173 \quad 178$

5 C S Sander, Technical Report No G558-10, Stanford University, Stanford, CA, U S A (1980)

$6 \mathrm{~K} \cdot \mathrm{W}$ Lee and K D Wise, IEEE Trans Electron Devices, ED-29 (1982) 34 - 41

$7 \mathrm{~K}$ Yamada, M Nishihara, R Kanzawa and R Kobayashı, Sensors and Actuators, 4 (1983) $63 \cdot 69$

8 S K Clark and K D Wise, IEEE Trans Electron Devices, ED-26 (1979) 1887 - 1896

9 A J Zuckerwar, J Acoust Soc Am, 64 (1978) $1278-1285$

10 C W Reedık, $U S$ Patent No 3436492,1 April 1969, filed 17 Jan 1966

11 A Yoshikawa and T Suzukı, $J$ Acoust Soc Am, 64 (1978) $725-730$

$12 \mathrm{~J}$ A Voorthuyzen and P Bergveld, IEEE Trans Electron Devices, ED-32 (1985) $1185-1190$

13 S M Sze, Physics of Semiconductor Devices, Wiley, New York, 1969

14 G M Sessler and J E West, Proc 7th Congr on Acoustics, Budapest, 1971, 23 E1, pp $413-416$

\section{Biographies}

Ad Sprenkels recelved his MS degree in electrical engineering from the Twente University of Technology, Enschede, The Netherlands, in 1983 
Since then he has been working at the same University towards his Ph D degree

His principle research is focused on the development of a solid state microphone for use in hearing ands

Johannes Arle Voorthuyzen was born in Capelle a/d IJssel, The Netherlands, on April 22, 1959 He recelved the M S degree in electrical engineering from the Delft University of Technology, Delft, The Netherlands, in 1982 In the same year he joined the Bio-information Group, Department of Electrical Engineering, Twente University of Technology, where he is working towards his $\mathrm{Ph} \mathrm{D}$ degree His current research is focused on electromechanical sensors for biomedical applications

Plet Bergueld was born in Oosterwolde, the Netherlands, on January 26, 1940 He received the M S degree in electrical engineering (electronics) from Eindhoven University of Technology, Eindhoven, the Netherlands, in 1965, and the Ph D degree from Twente University of Technology, Enschede, the Netherlands, in 1973

Since 1965 he has been a member of the Bio-information Group, Department of Electrical Engineering, Twente University of Technology He is further a member of the Coordination Centre for Biomedical Engineering and a member of the Research Unit Sensors and Actuators of this University In 1981 he became a member of the Semiconductor Group of the Foundation for Fundamental Research on Matter in the Netherlands

The subject of his dissertation was the ion-sensitive field-effect transistor (ISFET) and the OSFET He is involved in research on electronic measuring and stimulating methods in physiological systems, with special attention on in vivo biosensors $\mathrm{He}$ lectures on biomedical instrumentation for graduate students of Twente University of Technology and medical personnel of affiliated hospitals

In 1984 he was appointed as full professor in biosensor technology at the Twente University of Technology 